\title{
Actividad biológica del suelo de bosque templado en un transecto altitudinal, Parque Nacional Conguillío ( $38^{\circ} \mathrm{S}$ ), Chile
}

\author{
Soil biological activities of a temperate forests in altitudinal transect of Conguillío \\ National Park (38 S), Chile
}

\begin{abstract}
Alexis Lillo a, Hipólita Ramírez a , Francisco Reyes a, Nelson Ojeda b, Marysol Alvear a*
Autor correspondencia: ${ }^{a}$ Universidad de La Frontera, Facultad de Ingeniería, Ciencias y Administración, Departamento de Ciencias Químicas y Recursos Naturales. Temuco, Chile, tel.: 56-45-325438, fax 56-45-325440, malvear@ufro.cl

b Universidad de La Frontera, Facultad Ciencias Agropecuarias y Forestales, Departamento de Ciencias Forestales. Temuco, Chile.
\end{abstract}

SUMMARY

In Chile there are few studies describing the biological activities and soil nutrient dynamics in high mountain areas, therefore sensitive parameters able to express what happens in these ecosystems are required. The aim of this study was to determine soil biological properties through biochemical variables: fluorescein diacetate hydrolysis, carbon and nitrogen microbial biomass, $\beta$-glucosidase, carboximethylcellulase, acid phosphatase, urease and arylsulfatase; and chemical characteristics of soil in an altitudinal transect in Conguillío National Park ( $38^{\circ} \mathrm{S}$ ), Chile. We investigated four forest communities from 1,140 to 1,700 m a.s.l.: Nothofagus antarctica, Nothofagus alpina with Nothofagus dombeyi, Araucaria araucana with Nothofagus pumilio and Nothofagus pumilio (Krummholz). Biochemical parameters were sensitive to changes in altitude, obtaining the highest biological activities (fluorescein diacetate hydrolysis, nitrogen microbial biomass, acid phosphatase, $\beta$-glucosidase) in $N$. alpina with $N$. dombeyi (74.9 $\mu \mathrm{g}$ fluorescein $\mathrm{g}^{-1} \mathrm{~h}^{-1} ; 50.3 \mathrm{mg} \mathrm{N} \mathrm{kg}^{-1} ; 78.8 \mu \mathrm{mol} \mathrm{p}$-nitrophenol $\mathrm{g}^{-1} \mathrm{~h}^{-1} ; 9.14 \mu$ mol p-nitrophenol $\mathrm{g}^{-1} \mathrm{~h}^{-1}$, respectively) located at $1,250 \mathrm{~m}$ a.s.l. due to favorable climatic conditions, followed by $N$. antarctica and A. araucana with $N$. pumilio. The lowest levels of the biological activities are shown by $N$. pumilio (Krummholz) $\left(23.7 \mu\right.$ g fluorescein g-1 $\mathrm{h}^{-1} ; 3.1 \mathrm{mg} \mathrm{N} \mathrm{kg}^{-1} ; 6.2 \mu \mathrm{mol}$ p-nitrophenol g-1 $\mathrm{h}^{-1} ; 0.78 \mu \mathrm{mol}$ p-nitrophenol $\mathrm{g}^{-1} \mathrm{~h}^{-1}$, respectively) at $1,700 \mathrm{~m}$ a.s.l., located in restricted soil and climatic conditions.

Key words: soil microbial biomass, enzymatic activities, temperate forest.

\section{RESUMEN}

Existen pocos estudios en Chile que describen la actividad biológica y la dinámica de nutrientes en el suelo de las zonas de alta cordillera, por lo que se requiere incorporar parámetros que sean sensibles y expresen lo que sucede en este tipo de ecosistemas. El objetivo del presente estudio fue determinar las propiedades bioquímicas del suelo: hidrólisis de la fluoresceína diacetato, carbono y nitrógeno en la biomasa microbiana, $\beta$-glucosidasa, carboximetilcelulasa, fosfatasa ácida, ureasa y arilsulfatasa, y las características químicas del suelo en un transecto altitudinal en el Parque Nacional Conguillío (38 S), Chile. Se investigaron cuatro situaciones de comunidades boscosas (1.140 a 1.700 m s.n.m.): Nothofagus antarctica, Nothofagus alpina con Nothofagus dombeyi, Araucaria araucana con Nothofagus pumilio, y Nothofagus pumilio (Krummholz). Los parámetros bioquímicos fueron sensibles a las variaciones de altitud, registrándose los mejores índices de actividad biológica (hidrólisis de la fluoresceína diacetato, nitrógeno de la biomasa microbiana, fosfatasa ácida, $\beta$-glucosidasa) en $N$. alpina con $N$. dombeyi ubicado a $1.250 \mathrm{~m} \mathrm{s.n.m.} \mathrm{(74,9} \mu \mathrm{g}$ fluoresceína $\mathrm{g}^{-1} \mathrm{~h}^{-1} ; 50,3 \mathrm{mg}$ nitrógeno $\mathrm{kg}^{-1} ; 78,8 \mu \mathrm{mol}$-nitrofenol $\mathrm{g}^{-1} \mathrm{~h}^{-1} ; 9,14 \mu \mathrm{mol}$ p-nitrofenol $\mathrm{g}^{-1} \mathrm{~h}^{-1}$, respectivamente), debido a condiciones climáticas más favorables, seguidos de $N$. antarctica y A. araucana con $N$. pumilio. Los niveles más bajos de la actividad biológica se ubica en $N$. pumilio (Krummholz) a $1.700 \mathrm{~m}$ s.n.m. $\left(23,7 \mu \mathrm{g}\right.$ fluoresceína $\mathrm{g}^{-1} \mathrm{~h}^{-1} ; 3,1 \mathrm{mg}$ nitrógeno kg-1 ; $6,2 \mu \mathrm{mol} \mathrm{p}$-nitrofenol $\mathrm{g}^{-1} \mathrm{~h}^{-1} ; 0,78 \mu \mathrm{mol}$ p-nitrofenol $\mathrm{g}^{-1} \mathrm{~h}^{-1}$, respectivamente), situada en condiciones edafoclimáticas más restringidas.

Palabras clave: biomasa microbiana del suelo, actividades enzimáticas, bosques templados.

\section{INTRODUCCIÓN}

Los suelos del centro-sur de Chile han sido forjados durante su historia por una alta actividad volcánica, integrando en su formación gran cantidad de material eruptivo, en especial ceniza volcánica (Besoain 1985). Esta zona de Los Andes chilenos se caracteriza por presentar bosques templados dominados por varias especies caducifolias del género Nothofagus y por coníferas milenarias. La alta variabilidad ecofisiográfica presente en la región está influenciada, principalmente, por las condiciones climáticas y topográficas. Así, las variaciones latitudinal, altitudinal y longitudinal de las temperaturas y precipitaciones afectan los patrones de distribución de la vegetación (Luebert y Pliscoff 2006). La altitud es un factor crítico en la composición boscosa y, en conjunto con 
la humedad del suelo, son las variables ambientales más importantes que explican la distribución de comunidades vegetales (Velásquez 1994).

Aun cuando las condiciones abióticas se han interpretado tradicionalmente como los reguladores de la composición en la vegetación, las interacciones bióticas, particularmente en el suelo, también son reportadas como uno de los principales factores de la composición de las comunidades vegetales (Rodríguez-Loinaz et al. 2008). Los microorganismos del suelo representan el componente esencial del sistema biótico en los bosques naturales, determinantes en los ciclos biogeoquímicos de los nutrientes. La biota del suelo está influenciada por la calidad y cantidad de material vegetal aportado al suelo y por las características climáticas que también afectan la abundancia microbiana, las especies involucradas y su composición trófica (Wright y Coleman 2000).

Los parámetros bioquímicos son excelentes bioindicadores de la calidad y sanidad del suelo (Alvear et al. 2007ab) relacionados con los ciclos biogeoquímicos de los elementos (carbono, nitrógeno y fósforo). Uno de los parámetros importantes para determinar el tamaño de la actividad biológica en el suelo es la hidrólisis de la fluoresceína diacetato, que da cuenta de la microbiota activa en un determinado suelo (Green et al. 2006). El carbono y el nitrógeno en la biomasa microbiana determinan el tamaño aproximado de la comunidad de los microorganismos presente en el suelo. En tanto las actividades enzimáticas son cruciales en la disponibilidad de nutrientes para las plantas y microorganismos del suelo, y pueden ser útiles como indicadores en la diversidad funcional del suelo (Alef y Nannipieri 1995, Alvear et al. 2007ab). Entre estas enzimas están la $\beta$-glucosidasa, involucrada en la degradación de la celulosa (Turner et al. 2002) a su forma más simple; la carboximetilcelulasa, enmarcada dentro de las endocelulasas, enzimas capaces de hidrolizar enlaces glucosídicos al interior de las moléculas cristalinas de celulosa; la enzima ureasa, esencial en la transformación de urea en el suelo a amonio y dióxido de carbono, en tanto la actividad fosfatasa ácida cumple el rol de hidrolizar fósforo orgánico presente en el suelo, y la arilsulfatasa participa en el proceso de hidrólisis del éster-sulfato de la materia orgánica del suelo liberando azufre como ión sulfato $\left(\mathrm{SO}_{4}^{-2}\right)$.

En Chile son escasos los estudios biológicos y bioquímicos del suelo en comunidades boscosas de la zona andina. Lo anterior constituye una debilidad importante por ser indicadores sensibles de los cambios de uso de suelo, considerando los nuevos desafíos de manejo sustentable de los recursos renovables en el centro-sur de Chile (Frank y Finckh 1997, Huygens et al. 2005, Rivas et al. 2009). Una mejor comprensión de las funciones de la actividad enzimática del suelo de un ecosistema natural permite realizar una evaluación integrada de éste. Ello debido al papel crucial en varias actividades biológicas que cumplen en el suelo, a su facilidad de medición y a su respuesta rápida a las alteraciones producidas por el cambio de uso y manejo del suelo (Makoi y Kdakidemi 2008). En la Cordillera de Los Andes la vegetación boscosa se distribuye en un gradiente edafoclimático altitudinal, regulado fundamentalmente por las bajas temperaturas, la precipitación y depósitos recientes en el suelo producto del volcanismo acentuado, donde existen estrategias específicas de la biota, bajo condiciones restrictivas de nutrientes. Por ello, la actividad biológica y la descomposición de la materia orgánica del suelo debieran ser mínimas en el tiempo a mayor altitud. El objetivo central de este estudio es determinar la actividad biológica relacionada al suelo y la influencia de las propiedades químicas bajo distintas comunidades arbóreas en un transecto altitudinal en el Parque Nacional Conguillío.

\section{MÉTODOS}

Área de estudio. El estudio se realizó en el Parque Nacional Conguillío, ubicado en región de La Araucanía, $38^{\circ} 40^{\prime} \mathrm{S}$ y $71^{\circ} 45^{\prime} \mathrm{O}$, en la precordillera de Los Andes, Chile. Presenta un clima frío, húmedo y templado con meses secos en verano. La precipitación media anual para el área es de 2.500 a $3.000 \mathrm{~mm}$ (Pollmann 2003). La temperatura media anual es $8,6{ }^{\circ} \mathrm{C}$ con una media de $15,1{ }^{\circ} \mathrm{C}$ para enero (mes más cálido) y $1,9{ }^{\circ} \mathrm{C}$ para julio (mes más frío). Presenta precipitaciones de nieve desde mayo a septiembre. En cuanto a la temperatura y al régimen de lluvias, la zona pertenece al piso bioclimático mesotemplado y ombrotipo húmedo. El relieve del parque y su paisaje, en general, están influenciados por el volcán Llaima (3.125 m s.n.m.), actualmente activo. Debido a la permanente actividad volcánica en el sector, los suelos derivan de la descomposición de rocas volcánicas depositadas recientemente, toba y cenizas sobre los depósitos basálticos (Peralta 1975), originando, por lo general, suelos profundos y de drenaje rápido en las partes altas y lomajes suaves.

Vegetación en el Parque Nacional Conguillío. La vegetación está dominada por bosques mixtos de Nothofagus spp. con Araucaria araucana (Mol.) K. Koch (araucaria) y bosques de transición hacia el límite vegetacional. De acuerdo a la clasificación de Gajardo (1994), el parque se circunscribe en dos regiones ecológicas. La primera corresponde al bosque andino-patagónico, subregión de las cordilleras de La Araucanía, con la formación vegetal bosque caducifolio alto-andino con araucaria. La segunda es el bosque caducifolio, subregión del bosque caducifolio andino, con formación vegetal de bosque caducifolio mixto de la cordillera andina.

Nothofagus antarctica (G. Forst.) Oerst. (ñirre) presenta la mayor amplitud ecológica en su género, colonizando todo tipo de biótopos extremos que están vedados al resto de las especies del género (Ramírez et al. 1985). Esta especie se encuentra en el parque en la zona 
de cota inferior en una área tipificada como "bolsón de aire frío", colonizando ambientes con limitada vegetación y con escasa regeneración, formando bosques puros o bien bosques mixtos con A. araucana.

Nothofagus alpina (Poepp. et Endl.) Oerst. (raulí) se desarrolla en el parque entre los 900 y 1.300 m s.n.m. (Pollmann 2003), formando bosques puros con Nothofagus dombeyi (Mirb.) Oerst. (coihue). Pollmann (2001) clasifica a la asociación fitosociológica de ambas especies como Nothofagetum dombeyi-alpinae, perteneciendo al tipo forestal roble-raulí-coihue (Donoso 1981). Aquí, N. alpina domina en el estrato arbóreo respecto a $N$. dombeyi, pero sólo en condiciones de humedad y temperatura favorables, por ser menos resistente al frío que otros congéneres (Weinberger y Ramírez 2001).

Por su parte, A. araucana es una conífera que se desarrolla en altas cumbres hasta el límite vegetacional y, según la clasificación de Donoso (1981), corresponde al tipo forestal araucaria. Las condiciones óptimas de crecimiento están entre 1.300 y 1.600 m de altitud, donde se asocia con Nothofagus pumilio (Poepp. et Endl.) Krasser (lenga) en el dosel inferior. En ausencia de perturbaciones masivas, tanto A. araucana como N. pumilio siguen una dinámica de regeneración favorecida por la formación de claros, que conduce a estructuras multietáneas de ambas especies (Luebert y Pliscoff 2006).

La formación achaparrada o Krummholz, según la clasificación de pisos vegetacionales descrita por Luebert y Pliscoff (2006) y Donoso (1981), califica dentro del llamado bosque caducifolio achaparrado templado andino de $N$. pumilio, creciendo entre 1.600 y 2.000 m s.n.m., marcando el límite altitudinal de la vegetación boscosa.

Colecta de suelo. Se realizó un diseño aleatorio estratificado, agrupado en cuatro áreas de vegetación arbórea homogénea, al subir en un transecto altitudinal en el sendero Sierra Nevada en la primavera del año 2007 (cuadro 1). Se recolectaron cinco muestras compuestas de suelo en forma aleatoria para cada una de las situaciones, previo despeje del material vegetal y hasta una profundidad de $15 \mathrm{~cm}$. Posteriormente fueron pasadas por un tamiz de $2 \mathrm{~mm}$ de abertura de malla, guardadas en bolsas de plástico isotérmicas y conservadas a $4{ }^{\circ} \mathrm{C}$ hasta su posterior análisis. Cada muestra se analizó por triplicado, expresando los resultados con base en suelo seco $\left(105^{\circ} \mathrm{C}\right)$.
Actividades microbianas en el suelo. Para la hidrólisis de la fluoresceína diacetato (FDA) se utilizó la metodología descrita por Alvear et al. (2007) y los resultados se expresaron como $\mu \mathrm{g}$ de fluoresceína $\mathrm{g}^{-1} \mathrm{~h}^{-1}$. El carbono en la biomasa microbiana (CBM) y el nitrógeno en la biomasa microbiana (NBM) se midieron según el procedimiento de fumigación-extracción de Vance et al. (1987). El flujo de nitrógeno asociado a la biomasa se determinó siguiendo la técnica colorimétrica de Joergensen y Brookes (1990).

Actividades enzimáticas en el suelo. Para la actividad carboximetilcelulasa (CMC-asa), se utilizó el método de Schinner y von Mersi (1990), empleando como sustrato carboximetilcelulosa; la actividad de la enzima se expresó en $\mu \mathrm{mol}$ de glucosa $\mathrm{g}^{-1} \mathrm{~h}^{-1}$. Las actividades $\beta$-glucosidasa, fosfatasa ácida y arilsulfatasa se evaluaron según Alvear et al. (2005), expresándose éstas como $\mu$ mol de p-nitrofenol (PNF) $\mathrm{g}^{-1} \mathrm{~h}^{-1}$. Para la actividad ureasa se utilizó la metodología descrita por Gil-Sotres et al. (1992) y se expresó en $\mu$ mol de $\mathrm{NH}_{3} \mathrm{~g}^{-1} \mathrm{~h}^{-1}$.

Análisis químico del suelo. Según la norma chilena descrita por Sadzawka et al. (2004), se realizaron los análisis de $\mathrm{pH}$, materia orgánica (MO), cationes intercambiables (calcio, magnesio, sodio, potasio y aluminio), fracciones disponibles de fósforo, potasio y azufre, saturación de aluminio y capacidad de intercambio catiónico efectiva (CICE). También se obtuvo el contenido de humedad del suelo.

Análisis estadístico. A las variables biológicas del suelo (hidrólisis de la fluoresceína diacetato, nitrógeno en la biomasa microbiana, carbono en la biomasa microbiana, $\beta$-glucosidasa, actividad carboximetilcelulasa, fosfatasa ácida, ureasa y arilsulfatasa) así como a las variables químicas del suelo ( $\mathrm{pH}, \mathrm{MO}$, saturación de aluminio, capacidad de intercambio catiónico efectiva y humedad), se les verificó el supuesto de normalidad. A estas variables se les realizó un ANOVA de un factor, con un nivel de significancia de $95 \%(P<0,05)$. Para la comparación entre los cuatro tipos de vegetación en el gradiente altitudinal, se utilizó la prueba de Tukey con un nivel de significancia del $95 \%$. Para determinar la interacción entre variables se usó la prueba de correlación de Pearson (variables biológicas, $\mathrm{pH}$, materia orgánica, humedad, saturación

Cuadro 1. Resumen del área de estudio en un transecto altitudinal del sendero Sierra Nevada, Parque Nacional Conguillío (38 S), Chile. Summary of the study area in an altitudinal transect in Sierra Nevada trail at Conguillío National Park, $\left(38^{\circ} \mathrm{S}\right)$, Chile.

\begin{tabular}{llccc}
\hline Situación & \multicolumn{1}{c}{$\begin{array}{c}\text { Especies arbóreas } \\
\text { dominantes }\end{array}$} & $\begin{array}{c}\text { Altitud } \\
(\mathrm{m} \mathrm{s.n.m.)})\end{array}$ & $\begin{array}{c}\text { Densidad total } \\
\left(\text { árboles ha }{ }^{-1}\right)\end{array}$ & $\begin{array}{c}\text { Cobertura de copas } \\
(\%)\end{array}$ \\
\hline Ñirre & Nothofagus antarctica & 1.140 & 190 & 50 \\
Raulí-coihue & N. alpina y N. dombeyi & 1.250 & 410 & 85 \\
Araucaria-lenga & Araucaria araucana y N. pumilio & 1.500 & 180 & 70 \\
Krummholz & N. pumilio & 1.700 & 90 & 20 \\
\hline
\end{tabular}


de aluminio, capacidad de intercambio catiónico efectiva y fósforo, potasio y azufre disponibles). Se realizó un análisis factorial para todas las variables biológicas, $\mathrm{pH}$, materia orgánica, humedad, saturación de aluminio y capacidad de intercambio catiónico efectiva para explorar si ellas mostraban tendencias en la varianza producto de la gradiente altitudinal. Un análisis de varianza también se llevó a cabo para poner a prueba las diferencias entre la vegetación en el análisis factorial; fue usado el factor 1 por explicar éste la mayor cantidad de variables (Bastida et al. 2008). El análisis estadístico se realizó con el programa Statgraphic plus 5.1 para Windows.

\section{RESULTADOS}

En las cuatro comunidades arbóreas estudiadas en el transecto altitudinal (cuadro 2) el $\mathrm{pH}$ no presentó diferencias significativas $(P>0,05)$, correspondiendo a suelos ligeramente ácidos. El contenido de materia orgánica presentó diferencias significativas sólo entre raulí-coihue y Krummholz $(P=0,005)$. La mayor cobertura vegetal (tanto en el estrato arbóreo como arbustivo) en raulí-coihue permite una mayor acumulación de materia orgánica en el suelo. La humedad fue significativamente menor en Krummholz respecto a las otras situaciones $(P<0,05)$, coincidiendo con una baja cobertura arbórea y una mayor proporción de partículas gruesas (escoria volcánica) en los horizontes superficiales, disminuyendo la capacidad de retención de agua en el suelo.
El fósforo disponible en el suelo fue similar en ñirre y raulí-coihue (cuadro 2), pero significativamente mayores $(P<0,001)$ que araucaria-lenga y Krummholz. Igual situación se observó para el potasio disponible, siendo en Krummholz significativamente menor $(P<0,001)$. El azufre disponible fue significativamente mayor $(P<0,001)$ en raulí-coihue.

Los cationes intercambiables fueron significativamente mayores en raulí-coihue, ñirre y araucaria-lenga respecto a Krummholz (cuadro 2). El calcio fue el catión más abundante de todos en cada una de las comunidades arbóreas. Del mismo modo, la capacidad de intercambio catiónico efectiva fue de un bajo índice en Krummholz. La saturación de aluminio fue significativamente mayor $(P<0,05)$ en Krummholz.

Biomasa microbiana. Las actividades biológicas presentaron diferencias estadísticamente significativas entre las distintas asociaciones vegetales (cuadro 3 ), especialmente entre raulí-coihue y Krummholz para hidrólisis de la fluoresceína diacetato y nitrógeno y carbono en la biomasa microbiana. La hidrólisis de la fluoresceína diacetato fue mayor en raulí-coihue que en araucaria-lenga $(P=0,007)$ y Krummholz $(P<0,001)$. Comportamiento similar tiene el nitrógeno en la biomasa microbiana, siendo mayor estadísticamente en raulí-coihue que en ñirre $(P=0,001)$, araucaria-lenga $(P=0,033)$ y Krummholz $(P<0,001)$. Tanto la hidrólisis de la fluoresceína diacetato como el nitrógeno en la biomasa microbiana, en ñi-

Cuadro 2. Propiedades químicas del suelo en un transecto altitudinal en el sendero Sierra Nevada, Parque Nacional Conguillío (38 S), Chile.

Chemical properties in an altitudinal transect in Sierra Nevada trail at Conguillío National Park (38 S), Chile (mean value and standard deviation).

\begin{tabular}{|c|c|c|c|c|}
\hline Variable & $\begin{array}{c}\text { Ñirre } \\
(1.140 \text { m s.n.m.) }\end{array}$ & $\begin{array}{c}\text { Raulí-coihue } \\
\text { (1.250 m s.n.m.) }\end{array}$ & $\begin{array}{l}\text { Araucaria-lenga } \\
(1.500 \mathrm{~m} \text { s.n.m.) }\end{array}$ & $\begin{array}{c}\text { Krummholz } \\
\text { (1.700 m s.n.m.) }\end{array}$ \\
\hline $\mathrm{pH}\left(\mathrm{H}_{2} \mathrm{O}\right)$ & $5,52 \pm 0,35 a$ & $5,70 \pm 0,22 a$ & $5,42 \pm 0,05 a$ & $5,78 \pm 0,28 a$ \\
\hline Materia orgánica (\%) & $13,8 \pm 2,4 a b$ & $27,6 \pm 16,2 a$ & $18,9 \pm 0,7 \mathrm{ab}$ & $5,1 \pm 2,1 b$ \\
\hline Humedad (\%) & $27,9 \pm 2,7 a$ & $34,8 \pm 8,8 a$ & $31,9 \pm 2,8 a$ & $16,1 \pm 4,8 b$ \\
\hline $\mathrm{P}\left(\mathrm{mg} \mathrm{kg}^{-1}\right)$ & $18 \pm 2,3 \mathrm{a}$ & $21 \pm 2,9 \mathrm{a}$ & $9,0 \pm 1,0 b$ & $4,0 \pm 1,2 \mathrm{c}$ \\
\hline $\mathrm{K}\left(\mathrm{mg} \mathrm{kg}^{-1}\right)$ & $113 \pm 7,3 b$ & $176 \pm 11,0 \mathrm{a}$ & $125 \pm 4,5 b$ & $74 \pm 3,4 \mathrm{c}$ \\
\hline $\mathrm{S}\left(\mathrm{mg} \mathrm{kg}^{-1}\right)$ & $10,2 \pm 1,10 b$ & $17,5 \pm 2,61 \mathrm{a}$ & $7,3 \pm 0,30 \mathrm{c}$ & $9,54 \pm 0,28 \mathrm{bc}$ \\
\hline Ca int $\left(\mathrm{cmol}+\mathrm{kg}^{-1}\right)$ & $7,8 \pm 1,0 \mathrm{~b}$ & $14,6 \pm 2,0 \mathrm{a}$ & $6,8 \pm 0,2 b$ & $1,0 \pm 0,1 \mathrm{c}$ \\
\hline Mg int $\left(\mathrm{cmol}+\mathrm{kg}^{-1}\right)$ & $1,3 \pm 0,03 b$ & $2,2 \pm 0,20 \mathrm{a}$ & $1,3 \pm 0,02 b$ & $0,3 \pm 0,04 c$ \\
\hline $\mathrm{Na}$ int $\left(\mathrm{cmol}+\mathrm{kg}^{-1}\right)$ & $0,15 \pm 0,01 \mathrm{a}$ & $0,17 \pm 0,03 a$ & $0,15 \pm 0,02 \mathrm{a}$ & $0,09 \pm 0,01 \mathrm{c}$ \\
\hline $\mathrm{K}$ int $\left(\mathrm{cmol}+\mathrm{kg}^{-1}\right)$ & $0,29 \pm 0,02 b$ & $0,45 \pm 0,05 a$ & $0,32 \pm 0,02 b$ & $0,19 \pm 0,02 c$ \\
\hline $\mathrm{Al}$ intercambiable $\left(\mathrm{cmol}+\mathrm{kg}^{-1}\right)$ & $0,05 \pm 0,002 \mathrm{a}$ & $0,06 \pm 0,003 c$ & $0,11 \pm 0,002 \mathrm{a}$ & $0,08 \pm 0,002 b$ \\
\hline Saturación de $\mathrm{Al}(\%)$ & $0,5 \pm 0,21 c$ & $0,34 \pm 0,46 \mathrm{~d}$ & $1,3 \pm 0,03 b$ & $5,04 \pm 0,03 a$ \\
\hline CICE $\left(\mathrm{cmol}+\mathrm{kg}^{-1}\right)$ & $9,6 \pm 1,0 \mathrm{~b}$ & $17,4 \pm 2,0 \mathrm{a}$ & $8,7 \pm 0,2 b$ & $1,6 \pm 0,1 c$ \\
\hline Suma de bases $\left(\mathrm{cmol}^{+} \mathrm{kg}^{-1}\right)$ & $9,5 \pm 0,9 b$ & $17,4 \pm 1,8 \mathrm{a}$ & $8,6 \pm 0,1 b$ & $1,5 \pm 0,1 c$ \\
\hline
\end{tabular}

Valores corresponden al promedio y su respectiva desviación estándar. Letras distintas indica medias estadísticamente diferentes $(P<0,05)(\mathrm{n}=$ 20). P: fósforo disponible; K: potasio disponible; S: azufre disponible; Ca int: calcio intercambiable; $\mathrm{Mg}$ int: magnesio intercambiable; Na int: sodio intercambiable; $\mathrm{K}$ int: potasio intercambiable; CICE: capacidad de intercambio catiónico efectiva. 
Cuadro 3. Análisis de la biomasa microbiana y las actividades enzimáticas en un transecto altitudinal en el sendero Sierra Nevada, Parque Nacional Conguillío (38º S), Chile.

Analysis of microbial biomass and enzyme activities in an altitudinal transect in Sierra Nevada trail at Conguillío National Park ( $\left.38^{\circ} \mathrm{S}\right)$, Chile (mean value and standard deviation). Different letters indicate statistically different means, $P<0.05$.

\begin{tabular}{|c|c|c|c|c|}
\hline Variable & $\begin{array}{c}\text { Ñirre } \\
\text { (1.140 m s.n.m.) }\end{array}$ & $\begin{array}{c}\text { Raulí-coihue } \\
\text { (1.250 m s.n.m.) }\end{array}$ & $\begin{array}{l}\text { Araucaria-lenga } \\
\text { (1.500 m s.n.m.) }\end{array}$ & $\begin{array}{c}\text { Krummholz } \\
\text { (1.700 m s.n.m.) }\end{array}$ \\
\hline FDA $\left(\mu \mathrm{g}\right.$ fluoresceína $\left.\mathrm{g}^{-1} \mathrm{~h}^{-1}\right)$ & $56,3 \pm 9,0 \mathrm{ab}$ & $74,9 \pm 17,4 a$ & $45,6 \pm 11,8 b$ & $23,7 \pm 6,2 c$ \\
\hline $\mathrm{NBM}\left(\mathrm{mg} \mathrm{N} \mathrm{kg}{ }^{-1}\right)$ & $23,7 \pm 6,5 b$ & $50,3 \pm 15,4 a$ & $33,0 \pm 2,8 b$ & $3,1 \pm 5,5 c$ \\
\hline $\mathrm{CBM}\left(\mathrm{mg} \mathrm{C} \mathrm{kg}{ }^{-1}\right)$ & $1.154 \pm 91 \mathrm{ab}$ & $1.263 \pm 208 a$ & $1.048 \pm 123 \mathrm{ab}$ & $920 \pm 124 b$ \\
\hline$\beta$-glucosidasa $\left(\mu \mathrm{mol} \mathrm{PNF} \mathrm{g} \mathrm{g}^{-1} \mathrm{~h}^{-1}\right)$ & $3,52 \pm 0,41 b$ & $9,14 \pm 2,17 \mathrm{a}$ & $3,38 \pm 0,17 b$ & $0,78 \pm 0,44 \mathrm{c}$ \\
\hline CMC-asa $\left(\mu \mathrm{mol}\right.$ glucosa $\left.\mathrm{g}^{-1} \mathrm{~h}^{-1}\right)$ & $1,41 \pm 0,25 a$ & $1,01 \pm 0,42 \mathrm{ab}$ & $0,86 \pm 0,38 a b$ & $0,42 \pm 0,29 b$ \\
\hline Ureasa $\left(\mu \mathrm{mol} \mathrm{NH} \mathrm{N}_{3} \mathrm{~g}^{-1} \mathrm{~h}^{-1}\right)$ & $3,35 \pm 0,52 \mathrm{ab}$ & $4,54 \pm 0,87 a$ & $4,47 \pm 0,24 a$ & $2,09 \pm 0,90 \mathrm{~b}$ \\
\hline Fosfatasa ácida ( $\mu$ mol PNF g-1 $\left.\mathrm{h}^{-1}\right)$ & $32,6 \pm 9,4 b$ & $78,8 \pm 23,7 a$ & $42,7 \pm 8,0 b$ & $6,2 \pm 3,8 c$ \\
\hline Arilsulfatasa $\left(\mu \mathrm{mol} \mathrm{PNF} \mathrm{g}^{-1} \mathrm{~h}^{-1}\right)$ & $0,48 \pm 0,13 \mathrm{ab}$ & $0,42 \pm 0,08 \mathrm{ab}$ & $0,52 \pm 0,03 a$ & $0,32 \pm 0,09 b$ \\
\hline
\end{tabular}

Valores corresponden al promedio y su respectiva desviación estándar. Letras distintas indica medias estadísticamente diferentes $(P<0,05)$ $(\mathrm{n}=20)$. FDA: hidrólisis fluoresceína diacetato; NBM: nitrógeno en la biomasa microbiana; CBM: carbono en la biomasa microbiana; PNF: p-nitrofenol; CMC-asa: carboximetilcelulasa.

rre y araucaria-lenga, no presentaron diferencias significativas entre éstas, pero sí en comparación a Krummholz $(P<0,05)$. El contenido de carbono en la biomasa microbiana no reveló diferencias significativas $(P>0,05)$ entre ñirre, raulí-coihue y araucaria-lenga, en tanto Krummholz presentó un menor contenido con diferencias significativas sólo con raulí-coihue $(P<0,008)$.

Actividades enzimáticas. Las enzimas evaluadas presentaron distintos niveles de actividad en las comunidades arbóreas estudiadas (cuadro 3), registrándose los menores valores en Krummholz. Las actividades fosfatasa ácida y $\beta$-glucosidasa fueron significativamente mayores en raulícoihue ( $P<0,01$ y $P<0,001$, respectivamente), siendo altamente sensibles a los distintos tipos de vegetación. La actividad carboximetilcelulasa no tuvo diferencias significativas entre ñirre, raulí-coihue y araucaria-lenga, el índice más bajo se presentó en Krummholz $(P=0,002)$. La actividad ureasa fue mayor en raulí-coihue y araucaria-lenga, existiendo diferencias significativas entre estas asociaciones $(P<0,001)$ con Krummholz. La enzima arilsulfatasa fue mayor en araucaria-lenga que en Krummholz $(P=0,016)$.

Correlaciones entre las variables químicas y biológicas. La humedad y la materia orgánica del suelo tuvieron una correlación positiva con la mayoría de las actividades biológicas (cuadro 4), en especial con la hidrólisis de la fluoresceína diacetato, el nitrógeno en la biomasa microbiana, la $\beta$-glucosidasa, la ureasa y la fosfatasa ácida $(P<0,001)$. Entre las variables biológicas evaluadas la $\beta$-glucosidasa y la fosfatasa ácida muestran la más alta correlación positiva. La hidrólisis de la fluoresceína diacetato se correlacionó positivamente con la mayoría de las variables biológicas a excepción de la arilsulfatasa. El nitrógeno en la biomasa microbiana se correlacionó fuertemente con la fosfatasa ácida, $\beta$-glucosidasa y ureasa. El carbono en la biomasa microbiana tuvo una positiva correlación con las enzimas involucradas en el ciclo del carbono. La saturación de aluminio se correlacionó negativamente con la mayoría de los indicadores bioquímicos del suelo, especialmente, con la fluoresceína diacetato y el nitrógeno en la biomasa microbiana, como también para las variables materia orgánica, fósforo y potasio disponibles en el suelo. La capacidad de intercambio catiónico efectiva tuvo una alta correlación positiva con las variables fluoresceína diacetato, nitrógeno en la biomasa microbiana, $\beta$-glucosidasa y fosfatasa ácida $(P<0,001)$. El pH del suelo no se relacionó con las variables biológicas evaluadas, a excepción de arilsulfatasa, correlacionada negativamente.

Relación entre las variables biológicas y químicas con la vegetación. El análisis factorial arrojó tres factores principales que explicaron un $84,8 \%$ de la varianza, donde los factores 1,2 y 3 explicaron un $65,3 \%, 11,5 \%$ y $8 \%$, respectivamente (cuadro 5, figura 1). Las variables que tuvieron mayor incidencia en el factor 1 fueron el nitrógeno en la biomasa microbiana y las enzimas fosfatasa ácida y $\beta$-glucosidasa, así como también las variables materia orgánica, humedad y capacidad de intercambio catiónico efectiva. En tanto, en el factor 2 la mayor incidencia la ocupó la actividad carboximetilcelulasa y seguida, en menor medida, por el carbono en la biomasa microbiana, en tanto la saturación de aluminio lo hizo de forma negativa con un peso de -0,63. En el factor 3, las únicas variables con incidencia sobre ésta fueron la arilsulfatasa y, de manera negativa, el $\mathrm{pH}$ con un peso de $-0,83$. El factor 1 registró diferencias significativas $(P<0,05)$ bajo las distintas condiciones vegetales del transecto altitudinal (cuadro 5), donde raulí-coihue se ubicó en la zona más positiva de este factor y Krummholz en la zona más negativa (figura 1). 


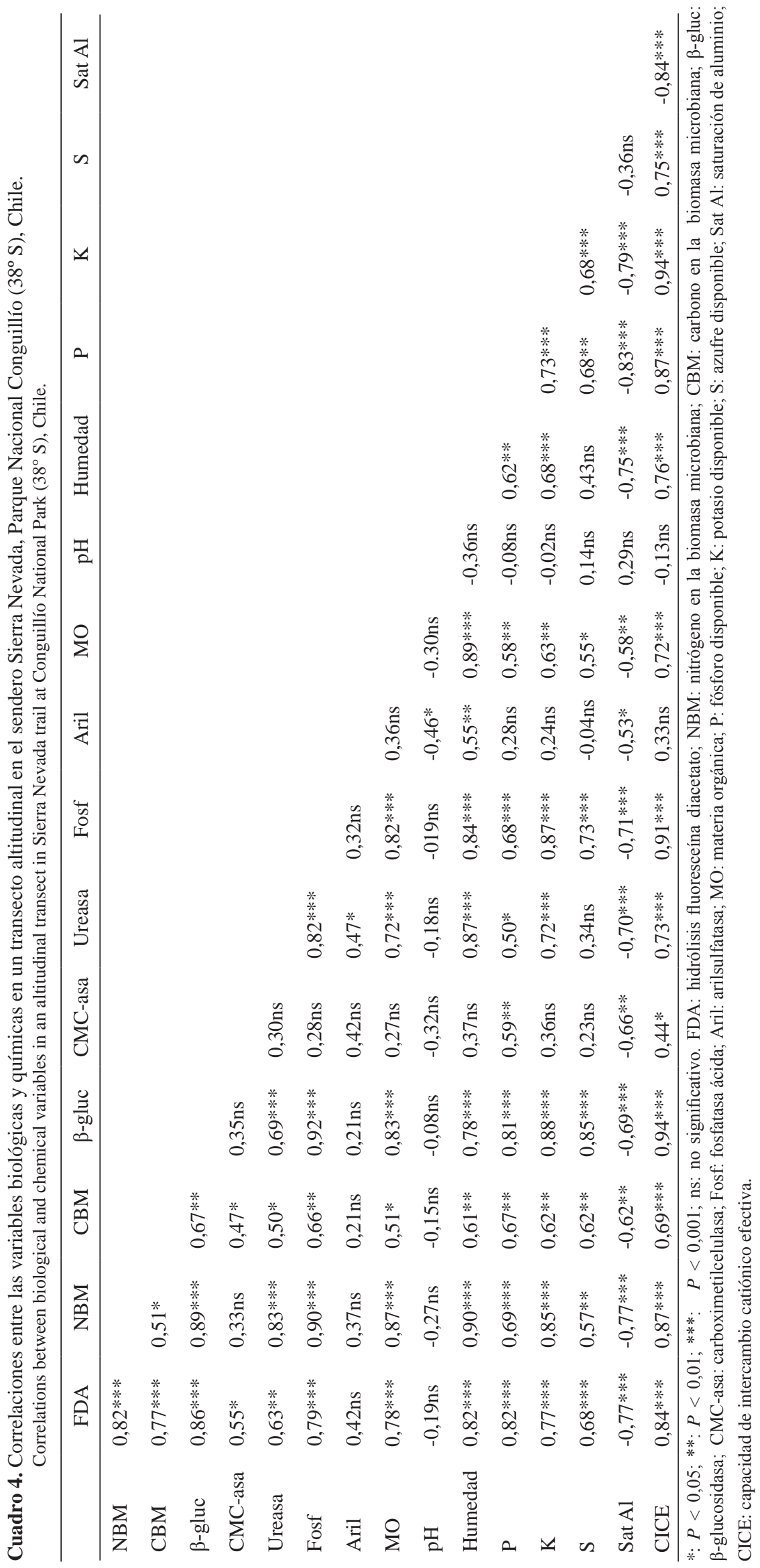


Cuadro 5. Análisis factorial para las variables biológicas y químicas, y ANOVA para el factor 1, en un transecto altitudinal en el sendero Sierra Nevada, Parque Nacional Conguillío (38 S), Chile.

Factor analysis for biological and chemical variables, and ANOVA of factor 1, for an altitudinal transect in Sierra Nevada trail of Conguillío National Park (38 S), Chile (different letters indicate means statistically different, $P<0.05$ ).

\begin{tabular}{|c|c|c|c|c|c|}
\hline \multicolumn{4}{|c|}{ Matriz de carga después de rotación varimax } & \multicolumn{2}{|l|}{ ANOVA Factor 1} \\
\hline & Factor 1 & Factor 2 & Factor 3 & Ñirre (1.140 m s.n.m.) & $\mathrm{b}$ \\
\hline FDA & 0,74 & 0,57 & 0,12 & Raulí-coihue (1.250 m s.n.m.) & $\mathrm{a}$ \\
\hline NBM & 0,92 & 0,19 & 0,21 & Araucaria-lenga (1.500 m s.n.m.) & $\mathrm{b}$ \\
\hline CBM & 0,52 & 0,67 & $-0,06$ & Krummholz (1.700 m s.n.m.) & c \\
\hline$\beta$-glucosidasa & 0,90 & 0,35 & $-0,07$ & & \\
\hline CMC-asa & 0,06 & 0,86 & 0,35 & & \\
\hline Ureasa & 0,83 & 0,13 & 0,27 & & \\
\hline Fosfatasa & 0,93 & 0,24 & 0,07 & & \\
\hline Arilsulfatasa & 0,23 & 0,23 & 0,79 & & \\
\hline MO & 0,88 & 0,09 & 0,24 & & \\
\hline $\mathrm{pH}$ & $-0,08$ & $-0,06$ & $-0,83$ & & \\
\hline Humedad & 0,86 & 0,21 & 0,39 & & \\
\hline Saturación aluminio & $-0,59$ & $-0,63$ & $-0,31$ & & \\
\hline CICE & 0,83 & 0,48 & 0,02 & & \\
\hline Varianza explicada (\%) & 65,3 & 11,5 & 8,0 & & \\
\hline Varianza explicada acumulada (\%) & 65,3 & 76,8 & 84,8 & & \\
\hline
\end{tabular}

Letras distintas indica medias estadísticamente diferentes $P<0,05$. FDA: hidrólisis fluoresceína diacetato; NBM: nitrógeno en la biomasa microbiana; CBM: carbono en la biomasa microbiana; CMC-asa: carboximetilcelulasa; MO: materia orgánica; CICE: capacidad de intercambio catiónico efectiva.

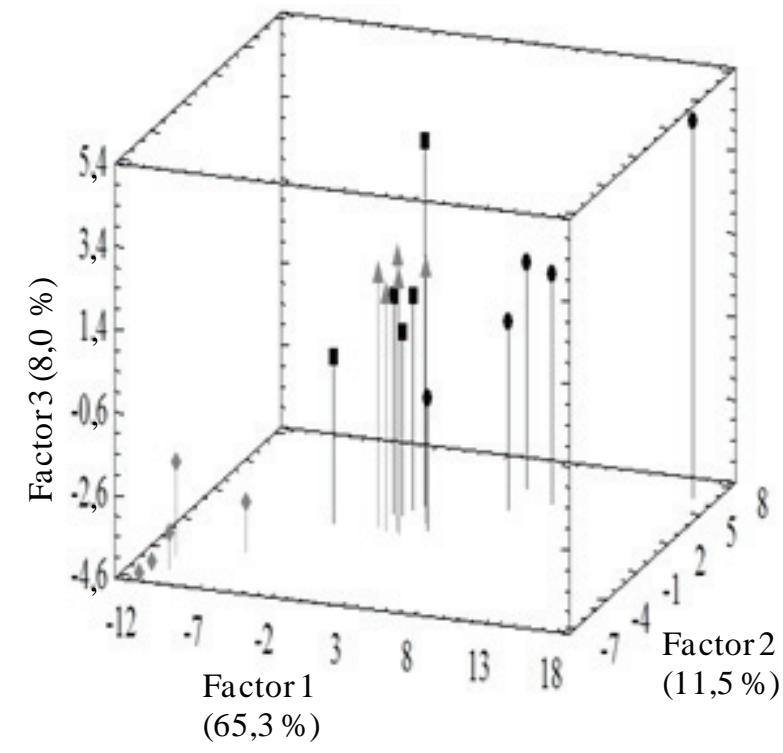

Figura 1. Dispersión de la vegetación según análisis factorial del estudio en un transecto altitudinal del sendero Sierra Nevada en el Parque Nacional Conguillío (38ㅇ S), Chile. $\mathbf{\square}$ : Nirre; raulí-coihue; $\mathbf{A}$ : araucaria-lenga; $\quad$ : Krummholz.

Dispersion of the vegetation according to factor analysis of an altitudinal transect study of the trail in Sierra Nevada, National Park Conguillío ( $\left.38^{\circ} \mathrm{S}\right)$, Chile.

\section{DISCUSIÓN}

Los niveles de actividad microbiana del suelo, en general, se encuentran por debajo de los rangos de actividad registrados en otros ecosistemas forestales de similar latitud (latizal de Nothofagus obliqua (Mirb)) (Alvear et al. 2007b). Sin embargo, la fosfatasa ácida tiene valores similares a los reportados por Rivas et al. (2007) en un bosque adulto de $N$. obliqua en la Depresión Intermedia (Paillaco $40^{\circ} 07^{\prime} \mathrm{S}$ ) en condiciones estacionales similares. En tanto, la actividad $\beta$-glucosidasa es mayor que los valores reportados por Alvear et al. (2007ab), estando en los rangos promedios a los encontrados por Trasar-Cepeda et al. (2000) en bosques de Querqus robur L. en Galicia, España. La actividad carboximetilcelulasa tiene valores inferiores a los mencionados por Alvear et al. (2007b), aunque cabe mencionar que no se cuenta con mayores registros de actividad para esta enzima junto a la arilsulfatasa, en ecosistemas boscosos naturales chilenos.

Actividad microbiana. Comparando cada una de las cuatro comunidades arbóreas, existe una marcada diferencia en las propiedades bioquímicas y químicas del suelo (cuadro 2 y 3), influenciadas directamente por los cambios de altitud, variando las condiciones ambientales en cada una de las áreas investigadas. Miralles et al. (2007) señalan a este respecto que las características altitudinales afectan la expresión de las propiedades bioquímicas del suelo. 
Una de las variables ambientales más importantes en la actividad biológica del suelo es la humedad, aumentando el crecimiento microbiano (Li y Sarah 2003, Abbasi et al. 2010), la que muestra, además, una correlación positiva con la mayoría de los parámetros biológicos (cuadro 4). La alta correlación positiva con la hidrólisis de la fluoresceína diacetato, indicaría una mejor disponibilidad de sustratos para los microorganismos del suelo ( $\mathrm{Li} \mathrm{y}$ Sarah 2003). El mayor tamaño de la población microbiana activa se encuentra en raulí-coihue, relacionado a los valores más altos de nitrógeno en la biomasa microbiana e hidrólisis de la fluoresceína diacetato, principalmente. En raulí-coihue se observa un alto contenido de materia orgánica en el suelo, producto de una mayor acumulación del material vegetal lábil aportado por $N$. dombeyi y, principalmente, $N$. alpina, que proporciona altos niveles de hojarasca durante las estaciones frías. Si bien los niveles de saturación de aluminio son bajos (cuadro 2), la correlación negativa con todas las variables biológicas, especialmente con la hidrólisis de la fluoresceína diacetato y el nitrógeno en la biomasa microbiana, implica una disminución en la actividad de los microorganismos del suelo (Krummholz) al aumentar la concentración de aluminio (Jones et al. 2001). Entre las situaciones ñirre y raulí-coihue, no manifiestan diferencias en el tamaño de la población microbiana en relación al carbono en la biomasa microbiana, lo que puede ser favorecido por los altos contenidos de carbono por la hojarasca de la especie decidua $N$. antarctica, durante el invierno en la primera situación, siendo inmovilizado por los microorganismos del suelo. Condición similar se manifiesta con araucarialenga, al no mantener diferencia con el carbono en la biomasa microbiana del suelo con las zonas más bajas, con aportes de compuestos carbonados lábiles desde $N$. pumilio, y en menor medida por A. araucana con aportes más recalcitrantes de carbono al suelo (Lusk 2001). Jia et al. (2005) mencionan que un bajo contenido de materia orgánica del suelo provoca una disminución del carbono en la biomasa microbiana, que queda demostrada en Krummholz, siendo significativamente menor a las otras tres situaciones.

Los niveles de nitrógeno en la biomasa microbiana son bastante sensibles a los cambios de la vegetación en el espectro altitudinal, siendo mayor en raulí-coihue diferenciándose claramente de las demás situaciones. En este sentido, se puede inferir que los aportes de nitrógeno provenientes del dosel superior para cada situación son diferentes. Staelens et al. (2005) registraron aportes de nitrógeno de la hojarasca de 56,3 y 46,9 $\mathrm{kg} \mathrm{ha}^{-1}$ año-1 $^{-1}$ en bosques de $N$. alpina y $N$. obliqua, respectivamente. El mayor índice de nitrógeno en la biomasa microbiana encontrado en raulí-coihue, es favorecido por los altos niveles de nitrógeno proveniente de la hojarasca de $N$. alpina, principalmente, pero aún importante son los aportes de $N$. dombeyi y el sotobosque acompañante. En la situación araucaria-lenga, el aporte de nitrógeno proviene básicamente de $N$. pumilio, ya que el aporte en hojarasca de A. araucana es menor por la alta longevidad de sus hojas de difícil descomposición y elevada relación carbono:nitrógeno (Lusk 2001). En el caso de ñirre el menor nivel de nitrógeno en la biomasa microbiana en comparación a raulí-coihue, correspondería al aporte casi exclusivo de $N$. antarctica que presenta, además, una escasa regeneración en este lugar (Ramírez et al. 1985). En la zona de mayor altitud el nitrógeno en la biomasa microbiana desciende significativamente respecto a las partes más bajas, claramente por el menor aporte de material vegetal de Krummholz al suelo y menor actividad biológica activa registrada (hidrólisis de la fluoresceína diacetato).

Actividad enzimática. La actividad de la $\beta$-glucosidasa y fosfatasa ácida presentan una alta sensibilidad a las distintas zonas de vegetación en el transecto altitudinal, siendo ambas bastante elevadas en raulí-coihue, influenciadas por el alto contenido de materia orgánica y humedad (cuadros 4 y 5), con fracciones más lábiles de la hojarasca, las que son fácilmente degradadas por estas enzimas; así también, la capacidad de intercambio catiónico efectiva como un buen indicador de la transferencia de nutrientes del suelo, mantiene un alto grado de interacción con las enzimas $\beta$-glucosidasa y fosfatasa ácida (cuadros 4 y 5), tal como lo señalan Senwo et al. (2007), destacándose, de esta manera, la importancia de la capacidad de intercambio catiónico efectiva en todo el transecto altitudinal.

A diferencia de la situación anterior, en Krummholz disminuye significativamente la actividad de estas enzimas, mostrándose la importancia del aporte de materia orgánica y humedad para la fosfatasa ácida (Críquet et al. 2004) y $\beta$-glucosidasa. En ñirre y araucaria-lenga los rangos de la $\beta$-glucosidasa son similares entre sí, aunque disminuyen un tercio respecto a raulí-coihue, lo que muestra una influencia clara a los cambios en la vegetación (Bastida et al. 2008) y a las condiciones climáticas presentes. A diferencia de Roose-Amsaleg et al. (2005) y Críquet et al. (2007), los altos niveles de fósforo disponible no necesariamente implica una retroinhibición sobre la actividad fosfatasa ácida, corroborado en este estudio por la correlación positiva entre ambas variables (cuadro 4), ya que al existir una mayor porción de fósforo orgánico lábil proveniente de la hojarasca en este tipo de bosques, aumenta la actividad de esta enzima, como lo señala Redel et al. (2008). El alto nivel de fosfatasa ácida en raulí-coihue pudo deberse a la estabilidad de la enzima por adsorción en la materia orgánica y el alto contenido de nitrógeno en el suelo (NBM) (Cárcamo et al. 2004), todas estas variables se relacionan entre sí, incidiendo positivamente en el factor 1 del análisis factorial (cuadro 5).

El proceso de degradación de la celulosa en el suelo, en general, es un proceso lento que depende de variables ambientales como la temperatura, contenido de agua, el pH, concentración de sustratos y el tipo de 
hojarasca. La enzima carboximetilcelulasa no manifiesta diferencias entre las tres primeras altitudes, sin mostrar una sensibilidad a los cambios de vegetación, lo que puede deberse a que la tasa de degradación de compuestos celulósicos provenientes del material vegetal es similar. La actividad carboximetilcelulasa aumenta su secreción por parte de los hongos durante la descomposición de hojarasca de carácter deciduo (Alef y Nannipieri 1995) y está relacionado al contenido de carbono en el suelo (Trasar-Cepeda et al. 2000). El menor nivel de actividad carboximetilcelulasa en Krummholz, se puede deber al bajo contenido de materia orgánica y a la mayor saturación de aluminio registrada en esta situación, indicado por la correlación negativa con esta enzima e incidiendo ambas en el factor 2 de forma contrapuesta (cuadro 5).

Los niveles de actividad ureasa manifiestan una similitud en la zona media del transecto, favorecidas por la acumulación de materia orgánica (cuadro 4), estabilizándose a los complejos órgano-minerales del suelo (Borie y Fuentealba 1982), siendo los complejos humus-ureasa resistentes a la desnaturalización por agentes proteolíticos o ambientales (Nannipieri et al. 1978), la actividad de esta enzima está íntimamente ligada a la cantidad de carbono orgánico presente en el suelo, e interactúa positivamente con este factor. El menor índice de actividad ureasa encontrado en Krummholz se puede deber, además de un bajo contenido de materia orgánica, a una condición de menor temperatura (Kissel et al. 1988), enzima que eleva su actividad al aumentar la temperatura (Makoi y Ndakidemi 2008).

La enzima arilsulfatasa es esencial para el ciclo del azufre, en particular en los suelos forestales (Fitzgerald y Strickland 1987). La actividad de esta enzima está fuertemente regulada por las plantas y la mayor masa radicular encontrada en las tres primeras altitudes, incorpora cantidades importantes de ácidos orgánicos por las raíces, considerado el sustrato más eficiente en promover esta enzima (Vong et al. 2003), por otro lado, el menor contenido de humedad en el suelo en Krummholz involucra una menor actividad de arilsulfatasa (Li y Sarah 2003). La enzima arilsulfatasa es sensible a cambios de $\mathrm{pH}$, lo que concuerda con lo señalado por Gupta et al. (1988), y que se muestra a su vez por el factor 3 (cuadro 5), lo que manifiesta que esta enzima se ve influenciada básicamente por el pH y de forma negativa (cuadro 4) en este estudio.

Las diferencias registradas por el factor 1 (cuadro 5), señala que las mejores condiciones ambientales se sitúan en raulí-coihue para la actividad microbiana y también vegetales, concordando con las características autoecológicas que presenta $N$. alpina, la que se desarrolla en suelos profundos y ricos en nutrientes (Pollmann 2003), encontrándose esta especie en un área bastante reducida en el transecto Sierra Nevada. En tanto ñirre y araucaria-lenga se concentran en la zona central (figura 1) de forma muy similar en relación al factor 1, lo que concuerda con las condiciones ambientales desfavorables en que se desarrolla $N$. antarctica (Ramírez et al. 1985), al encontrarse en un bolsón de aire frío; situación similar para araucaria-lenga, situada a $1.500 \mathrm{~m}$ s.n.m., con A. araucana dominando en conjunto con $N$. pumilio, bajo condiciones nutricionales más restrictivas. En tanto Krummholz, se encuentra en la parte negativa del factor 1 (figura 1), consecuencia de las condiciones ambientales extremas en que se sitúa, lo que genera los menores índices de actividad microbiana.

\section{CONCLUSIONES}

El gradiente altitudinal presente en la Cordillera de Los Andes manifiesta diferencias acentuadas en la distribución de las especies vegetales, variando a su vez en las actividades biológicas del suelo, dada las distintas características edafoclimáticas encontradas en cada situación del transecto altitudinal. Los niveles de actividad enzimática bajaron drásticamente en Krummholz, mientras que en raulí-coihue fueron, en general, mayores. Las enzimas $\beta$-glucosidasa y fosfatasa ácida junto al nitrógeno en la biomasa microbiana y la hidrólisis de la fluoresceína diacetato, fueron las actividades más sensibles al tipo de vegetación, influenciado por la materia orgánica y la humedad, principalmente.

\section{AGRADECIMIENTOS}

Proyectos DI-08-0045 y DIUFRO 120620, ambos de la Dirección de Investigación de la Universidad de La Frontera, Chile.

\section{REFERENCIAS}

Abbasi KM, M Zafar, T Sultan. 2010. Changes in soil properties and microbial indices across various management sites in the mountain environments of Azad Jammu and Kashmir. Communications in Soil Science and Plant Analysis 41(6): 768-782.

Alef K, P Nannipieri. 1995. Methods in Applied Soil Microbiology and Biochemistry. London, England. Academic Press. 576 p.

Alvear M, A Rosas, JL Rouanet, F Borie. 2005. Effects of three soil tillage systems on some biological activities in an Ultisol from southern Chile. Soil and Tillage Research 82: 195-202.

Alvear M, F Reyes, A Morales, C Arriagada, M Reyes. 2007. Actividad biológica y agregados estables al agua en dos tipos de formaciones vegetales de un bosque templado del Centro-Sur de Chile con perturbación antrópica. Ecología Austral 17: 113-122.

Alvear M, C Urra, R Huaiquilao, M Astorga, F Reyes. 2007. Actividades biológicas y estabilidad de agregados estables en un suelo del bosque templado chileno bajo dos etapas sucesionales y cambios estacionales. Revista de la Ciencia del Suelo y Nutrición Vegetal 7(3): 38-50.

Bastida F, GG Barberá, C García, T Hernández. 2008. Influence of orientation, vegetation and season on soil microbial and biochemical characteristics under semiarid conditions. 
Applied Soil Ecology 38: 62-70.

Besoain ME. 1985. Los suelos. In Tosso T ed. Suelos volcánicos de Chile. Santiago, Chile. INIA. p. 25-107.

Borie F, R Fuentealba. 1982. Bioquímica de suelos derivados de cenizas volcánicas. II. Actividad ureásica. Agricultura Técnica 42(2): 135-141.

Cárcamo A, L Puentes, R Godoy, C Oyarzún, E Valenzuela. 2004. Actividad biológica del suelo en un bosque de Nothofagus obliqua (Mirb.) Oerst., centro-sur de Chile. Revista de la Ciencia del Suelo y Nutrición Vegetal 4(2): 14-25.

Críquet S, AM Farnet, E Ferre, J Le Petit. 2004. Annual dynamics of phosphatase activities in an evergreen oak litter: influence of biotic and abiotic factors. Soil Biology and Biochemistry 36: 1111-1118.

Críquet S, A Braud, S Nèble. 2007. Short-term effects of sewage sludge application on phosphatase activities and available $\mathrm{P}$ fractions in Mediterranean soils. Soil Biology and Biochemistry 39(4): 921-929.

Donoso C. 1981. Tipos forestales de los bosques nativos de Chile. Documento de trabajo $\mathrm{N}^{\circ}$ 38. Santiago, Chile. FAOCONAF.

Fitzgerald JW, TC Strickland. 1987. Mineralization of organic sulfur in the $\mathrm{O}_{2}$ horizon of a hardwood forest: involvement of sulphatase enzymes. Soil Biology and Biochemistry 19: 779-781.

Frank D, M Finckh. 1997. Impactos de las plantaciones de pino oregón sobre la vegetación y el suelo en la zona centro-sur de Chile. Revista Chilena de Historia Natural 70: 191-211.

Gajardo R. 1994. La vegetación natural de Chile. Clasificación y distribución geográfica. Santiago, Chile. Univesitaria. 165 p.

Gil-Sotres F, M Trasar-Cepeda, C Ciardi, B Ceccantti, M Leirós. 1992. Biochemical characterization of biological activity in very young mine soils. Biology and Fertility of Soils 13: 25-30.

Green VS, DE Stott, M Diack. 2006. Assay for fluorescein diacetate hydrolytic activity: Optimization for soil samples. Soil Biology and Biochemistry 38(4): 693-701.

Gupta VVSR, JR Lawrence, JJ Germida. 1988. Impact of elemental sulfur fertilization on agricultural soils. I. Effects on microbial biomass and enzyme activities. Canadian Journal of Soil Science.

Huygens D, P Boeckx, O Van Cleemput, R Godoy, C Oyarzún. 2005. Aggregate structure and stability linked to carbon dinamic in a Chilean Andisol. Biogeosciences 2: 203-238.

Jia G, J Cao, C Wang, G Wang. 2005. Microbial biomass and nutrients in soil at the different stages of secundary forest succession in Ziwulin, northwest China. Forest Ecology and Management 217: 117-125.

Joergensen R, P Brookes. 1990. Ninhydrin-reactive measurements of microbial biomass in $0.5 \mathrm{M} \mathrm{K}_{2} \mathrm{SO}_{4}$ soil extracts. Soil Biology and Biochemistry 22: 1023-1028.

Kissel DE, ML Cabrera, RB Ferguson. 1988. Reactions of ammonia and urea hydrolysis products with soil. Soil Science Society of American Journal 52: 1793-1796.

Jones DL, T Eldhuset, HA De Wit, B Swensen. 2001. Aluminium effects on organic acid mineralization in a Norway spruce forest soil. Soil Biology and Biochemistry 33(9): 12591267.

Li X, P Sarah. 2003. Enzyme activities along a climatic transect in the Judean Desert. Catena 53: 349-363.

Luebert F, P Pliscoff. 2006. Sinopsis bioclimática y vegetacional de Chile. Santiago, Chile. Universitaria. p 316.

Lusk C. 2001. Leaf life spans of some conifers of the temperate forests of South America. Revista Chilena de Historia Natural 74: 711-718.

Makoi J, P Ndakidemi. 2008. Selected soil enzymes: Examples of their potential roles in the ecosystem. African Journal of Biotechnology 7(3): 181-191

Miralles I, R Ortega, M Sánchez-Marañón, MC Leirós, C TrasarCepeda, F Gil-Sotres. 2007. Biochemical properties of range and forest soils in Mediterranean mountain environments. Biology and Fertility Soil 43: 721-729.

Nannipieri P, B Ceccanti, S Cervelli, P Sequi. 1978. Stability and kinetic properties of humus-urease complexes. Soil Biology and Biochemistry 10: 143-147.

Peralta M. 1975. Ecología y silvicultura del bosque nativo chileno. Suelos. Facultad de Ciencias Forestales. Universidad de Chile, Santiago. 35 p. (Boletín Técnico Nº 31).

Pollmann W. 2001. Caracterización florísticas y posición sintaxonómica de los bosques caducifolios de Nothofagus alpina (Poepp. et Endl.) Oerst. en el centro-sur de Chile. Phytocoenologia 31: 353-400.

Pollmann W. 2003. Stand structure and dendroecology of an old-growth Nothofagus forest in Conguillio National Park, south Chile. Forest Ecology and Management 176: 87-103.

Rodríguez-Loinaz G, M Onaindia, I Amezaga, I Mijangos, C Garbisu. 2008. Relationship between vegetation diversity and soil functional diversity in native mixed-oak forests. Soil Biology and Biochemistry 40(1): 49-60.

Ramírez C, M Correa, H Figueroa; J San Martín. 1985. Variación del habito y hábitat de Nothofagus antarctica en el centro sur de Chile. Bosque 6(2): 55-73.

Redel Y, R Rubio, R Godoy, F Borie. 2008. Phosphorus fractions and phosphatase activity in an Andisol under different forest ecosystems. Geoderma 145: 216-221.

Rivas Y, R Godoy, E Valenzuela, J Leiva, C Oyarzún, M Alvear. 2007. Actividad biológica del suelo en dos bosques de Nothofagus del centro sur de Chile. Gayana Botánica 64: 81-92.

Rivas Y, C Oyarzún, R Godoy, E Valenzuela. 2009. Mineralización del nitrógeno, carbono y actividad enzimática del suelo en un bosque de Nothofagus obliqua (Mirb) Oerst y una plantación de Pinus radiata D. Don. del centro-sur de Chile. Revista Chilena de Historia Natural 82: 119-134.

Roose-Amsaleg C, P Mora, M Harry. 2005. Physical, chemical and phosphatase activities characteristics in soil-feeding termite nests and tropical rainforest soils. Soil Biology and Biochemistry 37(10): 1910-1917.

Sadzawka A, M Carrasco, R Grez, M Mora. 2004. Métodos de análisis recomendados para los suelos chilenos. Comisión de Normalización y Acreditación. Santiago, Chile. Sociedad Chilena de la Ciencia del Suelo. p 113.

SchinnerF, Wvon Mersi.1990. Xylanase, Carboxymethylcellulase and invertase activity in soil and improved method. Soil Biology and Biochemistry 16: 511-516.

Senwo Z, D Ranatunga, I Tazisong, R Taylor, Z He. 2007. Phosphatase activity of Ultisols and relationship to soil fertility indices. Journal of Food Agriculture and Environment 5: 262-266.

Staelens J, R Godoy, C Oyarzún, K Thibo, K Verheyen. 2005. Nitrogen fluxes in throughfall and litterfall in two 
Nothofagus forest in Southern Chile. Gayana Botanica 62(2): 63-71.

Trasar-Cepeda C, MC Leirós, F Gil-Sotres. 2000. Biochemical properties of acid soils under climax vegetation (Atlantic oakwood) in an area of the European temperate-humid zone (Galicia, NW Spain): specific parameters. Soil Biology and Biochemistry 32: 747-755.

Turner B, D Hopkins, P Haygarth, N Oestle. 2002. $\beta$-Glucosidase activity in pasture soils. Applied Soil Ecology 20: 157-162.

Velásquez A. 1994. Multivariate analysis of the vegetation of the volcanoes Tláloc and Pelado, Mexico. Journal of Vegetation Science 5: 263-270.
Vong PC, O Dedourge, F Lasserre-Joulin, A Guckert. 2003. Immobilized-S, microbial biomass-S and soil arylsulfatase activity in the rhizosphere soil of rape and barley as affected by labile substrate $\mathrm{C}$ and $\mathrm{N}$ additions. Soil Biology and Biochemistry 35: 1651-1661.

Weinberger P, C Ramírez. 2001. Sinecología de la regeneración natural del raulí (Nothofagus alpina) Fagaceae, Magnoliopsida. Bosque 22: 11-26.

Wright CJ, DC Coleman. 2000. Cross-site comparison of soil microbial biomass, soil nutrient status, and nematode trophic groups. Pedobiologia 44: 2-23.

Recibido: 07.05.10

Aceptado: 15.12.10 